\title{
SEQUENTIAL INTERVAL ESTIMATION OF A LOCATION PARAMETER WITH THE FIXED WIDTH IN THE UNIFORM DISTRIBUTION WITH AN UNKNOWN SCALE PARAMETER
}

\author{
Masafumi Akahira and Ken-ichi Koike \\ Institute of Mathematics, University of Tsukuba, \\ Tsukuba, Ibaraki 305-8571, Japan \\ E-mail: akahira@math.tsukuba.ac.jp, koike@math.tsukuba.ac.jp
}

Key words: Uniform distribution, location parameter, scale parameter, sequential interval estimation, stopping rule, asymptotic consistency.

AMS 2000 subject classification. Primary 62L12, Secondary 62F25.

\begin{abstract}
We consider a sequential interval estimation with fixed width of a location parameter $\theta$ of a sequence of uniform random variables with unknown scale $\xi$. A stopping rule is proposed and its asymptotic properties are investigated. Numerical evaluations are also done. Further, the exact distribution of the size of sample is given.
\end{abstract}




\section{INTRODUCTION}

Suppose that we are to estimate a location parameter $\theta$ of a sequence of random observations $X_{1}, X_{2}, \ldots, X_{n}, \ldots$ with unknown scale $\xi$. We would like to obtain sequentially a confidence interval of fixed width $d$ with confidence coefficient $1-\alpha$. Obviously we can not obtain a fixed sample size procedure if $\xi$ is unknown.

In regular cases, if the maximum likelihood estimator (MLE) $\hat{\theta}$ of $\theta$ is at least asymptotically independent of the MLE $\hat{\xi}$ of $\xi$, and asymptotically normally distributed with variance $\left(n I_{\xi}\right)^{-1}$, we can obtain an asymptotically valid procedure when $d$ is small by continuing sampling as long as

$$
\sqrt{n I_{\hat{\xi}_{n}}} u_{\alpha / 2}>d
$$

where $u_{\alpha / 2}$ is the upper $100(\alpha / 2)$ percentile, and stop sampling when $\sqrt{n I_{\hat{\xi}_{n}}} u_{\alpha / 2} \leq d$, and construct the interval $\left[\hat{\theta}_{n}-d, \hat{\theta}_{n}+d\right]$, where $I_{\xi}$ is the Fisher information on $\xi$. Here $\hat{\theta}_{n}$ and $\hat{\xi}_{n}$ are the MLEs based on $X_{1}, \ldots, X_{n}$. It may be necessary to prove the above statement rigorously with a set of regularity conditions, but anyway it is possible to prove the validity of the statement if we assume the appropriate set of regularity conditions, and we would not go further into the details of the proof. There are many works on the fixed-width interval estimation of normal mean (see, e.g. Ghosh et al. (1997)).

But in the non-regular case, it may happen that even asymptotically $\hat{\xi}_{n}$ and $\hat{\theta}_{n}$ are independent, then the above procedure would fail to be valid. In this paper, we consider the case of the uniform distribution on the interval $[\theta-(\xi / 2), \theta+(\xi / 2)]$, where $\theta$ and $\xi$ are unknown, and obtain sequentially a confidence interval of $\theta$ with fixed width $d$ and confidence coefficient $1-\alpha$. In the case of the uniform distribution on the interval $(0, \theta)$, a similar problem 
was studied by Graybill and Connell (1964), Cooke (1971), Akahira (1993), Bose (2001), Mukhopadhyay and Cicconetti (2002) and others. A sequential point estimation of $\theta$ of the uniform distribution $U(\theta-(1 / 2), \theta+(1 / 2))$ was also discussed by Wald (1950). A uniform distribution may be used as a model in some practical problems (see, e.g. Perruzzi and Hilliard (1984) and Zimmels (1983)).

\section{CONFIDENCE INTERVAL OF $\theta$}

Suppose that $X_{1}, X_{2}, \ldots, X_{n}, \ldots$ is a sequence of independent and identically distributed (i.i.d.) random variables according to the uniform distribution on the interval $[\theta-(\xi / 2), \theta+(\xi / 2)]$, where $\theta\left(\in \mathbb{R}^{1}\right)$ and $\xi(>0)$ are unknown.

Let $X_{(1)}:=\min _{1 \leq i \leq n} X_{i}, X_{(n)}:=\max _{1 \leq i \leq n} X_{i}$. Then the midrange and the range are

$$
M_{n}:=\frac{1}{2}\left(X_{(1)}+X_{(n)}\right), R_{n}:=X_{(n)}-X_{(1)},
$$

respectively. When a confidence coefficient $1-\alpha$ and a width $d(>0)$ are given, we want to determine $n$ such that

$$
P_{\theta, \xi}\left\{\left|M_{n}-\theta\right| \leq d\right\} \geq 1-\alpha
$$

Now we consider the conditional probability given $R_{n}$

$$
\begin{aligned}
P_{\theta, \xi}\left\{\frac{M_{n}-\theta}{R_{n}} \leq t \mid R_{n}\right\} & =P_{\theta, \xi}\left\{M_{n}-\theta \leq t R_{n} \mid R_{n}\right\} \\
& =P_{\theta, \xi}\left\{\frac{M_{n}-\theta}{\xi} \leq t \frac{R_{n}}{\xi} \mid \frac{R_{n}}{\xi}\right\}
\end{aligned}
$$

for $t \in \mathbb{R}^{1}$. Letting $Y_{i}:=\left(X_{i}-\theta\right) / \xi(i=1,2, \ldots, n, \ldots)$, we see that $Y_{1}, Y_{2}, \ldots, Y_{n}, \ldots$ is a sequence of i.i.d. random variables according to the 
uniform distribution on $[-1 / 2,1 / 2]$. Then $Y_{(1)}:=\min _{1 \leq i \leq n} Y_{i}=\left(X_{(1)}-\theta\right) / \xi$ and $Y_{(n)}:=\max _{1 \leq i \leq n} Y_{i}=\left(X_{(n)}-\theta\right) / \xi$. We also have

$$
\frac{M_{n}-\theta}{\xi}=\frac{1}{2}\left(Y_{(1)}+Y_{(n)}\right)=: M_{n}^{\prime}, \frac{R_{n}}{\xi}=Y_{(n)}-Y_{(1)}=: R_{n}^{\prime} .
$$

From (2.2) we obtain

$$
P_{\theta, \xi}\left\{M_{n}-\theta \leq t R_{n} \mid R_{n}\right\}=P_{\theta, \xi}\left\{M_{n}^{\prime} \leq t R_{n}^{\prime} \mid R_{n}^{\prime}\right\} .
$$

Lemma 2.1. The conditional probability of $M_{n}^{\prime}$ given $R_{n}^{\prime}=r$ is given by

$$
P_{\theta, \xi}\left\{M_{n}^{\prime} \leq t r \mid R_{n}^{\prime}=r\right\}= \begin{cases}0 & \text { for } t \leq-\frac{1}{2}\left(\frac{1}{r}-1\right) \\ \frac{1}{2}+\frac{t r}{1-r} & \text { for } \quad-\frac{1}{2}\left(\frac{1}{r}-1\right)<t \leq \frac{1}{2}\left(\frac{1}{r}-1\right), \\ 1 & \text { for } t \geq \frac{1}{2}\left(\frac{1}{r}-1\right) .\end{cases}
$$

Proof. Since the joint density of $M_{n}^{\prime}$ and $R_{n}^{\prime}$ is given by

$$
f_{M_{n}^{\prime}, R_{n}^{\prime}}(m, r)= \begin{cases}n(n-1) r^{n-2} & \text { for } 0<r<1,|r| \leq 1-2|m|, \\ 0 & \text { otherwise }\end{cases}
$$

it follows that the marginal density of $R_{n}^{\prime}$ is

$$
f_{R_{n}^{\prime}}(r)= \begin{cases}n(n-1) r^{n-2}(1-r) & \text { for } 0<r<1 \\ 0 & \text { otherwise }\end{cases}
$$

Hence the conditional density of $M_{n}^{\prime}$ given $R_{n}^{\prime}$ is

$$
f_{M_{n}^{\prime} \mid R_{n}^{\prime}}(m \mid r)= \begin{cases}\frac{1}{1-r} & \text { for } \quad|m| \leq \frac{1}{2}-\frac{r}{2} \\ 0 & \text { otherwise }\end{cases}
$$

where $0<r<1$. Note that this is the uniform density on the interval $\left[-(1 / 2)+\left(R_{n}^{\prime} / 2\right),(1 / 2)-\left(R_{n}^{\prime} / 2\right)\right]$. From $(2.7)$ we have for $|t| r \leq(1-r) / 2$

$$
P_{\theta, \xi}\left\{M_{n}^{\prime} \leq t r \mid R_{n}^{\prime}=r\right\}=\int_{-\frac{1}{2}+\frac{r}{2}}^{t r} \frac{1}{1-r} d m=\frac{1}{2}+\frac{t r}{1-r},
$$


and (2.4) for other cases. This completes the proof.

From (2.3) and Lemma 2.1 we have the following.

Theorem 2.1. For $t>0$, the conditional probability given $R_{n}$ is given by

$$
P_{\theta, \xi}\left\{\left|M_{n}-\theta\right| \leq t R_{n} \mid R_{n}\right\}= \begin{cases}\frac{t}{\frac{1}{2}\left(\frac{\xi}{R_{n}}-1\right)} & \text { for } \quad 0<t \leq \frac{1}{2}\left(\frac{\xi}{R_{n}}-1\right) \\ 1 & \text { for } \quad t>\frac{1}{2}\left(\frac{\xi}{R_{n}}-1\right)\end{cases}
$$

Proof. From (2.2) and Lemma 2.1 we have for $t>0$

$$
\begin{aligned}
& P_{\theta, \xi}\left\{\left|M_{n}-\theta\right| \leq t R_{n} \mid R_{n}\right\}=P_{\theta, \xi}\left\{\left|M_{n}^{\prime}\right| \leq t R_{n}^{\prime} \mid R_{n}^{\prime}\right\} \\
& = \begin{cases}\frac{t}{\frac{1}{2}\left(\frac{1}{R_{n}^{\prime}}-1\right)} & \text { for } \quad 0<t \leq \frac{1}{2}\left(\frac{1}{R_{n}^{\prime}}-1\right), \\
1 & \text { for } \quad t>\frac{1}{2}\left(\frac{1}{R_{n}^{\prime}}-1\right)\end{cases} \\
& =\left\{\begin{array}{lll}
\frac{t}{\frac{1}{2}\left(\frac{\xi}{R_{n}}-1\right)} & \text { for } & 0<t \leq \frac{1}{2}\left(\frac{\xi}{R_{n}}-1\right), \\
1 & \text { for } \quad t>\frac{1}{2}\left(\frac{\xi}{R_{n}}-1\right) .
\end{array}\right.
\end{aligned}
$$

This completes the proof.

Letting $d=t R_{n}$ and

$$
\frac{d / R_{n}}{\frac{1}{2}\left(\frac{\xi}{R_{n}}-1\right)} \geq 1-\alpha
$$

we have from (2.2) and Theorem 2.1

$$
P_{\theta, \xi}\left\{\left|M_{n}-\theta\right| \leq d \mid R_{n}\right\} \geq 1-\alpha
$$

hence

$$
E_{\theta, \xi}\left[P_{\theta, \xi}\left\{\left|M_{n}-\theta\right| \leq d \mid R_{n}\right\}\right] \geq 1-\alpha .
$$

From (2.1), (2.8) and (2.9) we stop when

$$
\xi-R_{n} \leq \frac{2 d}{1-\alpha}
$$


but it is not useful because $\xi$ is unknown. Since

$$
E_{\theta, \xi}\left[\xi-R_{n}\right]=\frac{2 \xi}{n+1},
$$

it follows that

$$
E_{\theta, \xi}\left[\xi-R_{n}\right]=E\left[\frac{2}{n-1} R_{n}\right] .
$$

So, instead of $\xi-R_{n}$ in $(2.10)$, we use $2 R_{n} /(n-1)$. Hence, we stop when

$$
R_{n} \leq \frac{(n-1) d}{1-\alpha}
$$

and construct the confidence interval of $\theta$

$$
\left[M_{n}-d, M_{n}+d\right]
$$

that is, we consider the sequential estimation procedure $\left(\tau_{0},\left[M_{\tau_{0}}-d, M_{\tau_{0}}+\right.\right.$ $d]$ ), where $\tau$ is given by

$$
\tau_{0}=\tau\left(\frac{d}{1-\alpha}\right):=\inf \left\{\begin{array}{l|l}
n>1 & \frac{R_{n}}{n-1} \leq \frac{d}{1-\alpha}
\end{array}\right\}
$$

Note that this stopping rule is bounded with probability 1 since $0 \leq R_{n} \leq \xi$ with probability 1 and $(2.11)$, that is, $P_{\theta, \xi}\left(\tau_{0} \leq\left\lceil\frac{\xi(1-\alpha)}{d}\right\rceil+1\right)=1$ where $\lceil x\rceil$ means the least integer greater than or equal to $x$.

However, this sequential interval estimation procedure is not asymptotically consistent. So, instead of $\tau_{0}$, we consider another stopping rule:

$$
\tau_{1}=\tau\left(-\frac{2 d}{\log \alpha}\right):=\inf \left\{\begin{array}{l|l}
n \geq 2 & \frac{R_{n}}{n-1} \leq-\frac{2 d}{\log \alpha}
\end{array}\right\}
$$

Consider the interval estimation of $\theta$ when $\xi$ is known. Since the density function of $M_{n}^{\prime}$ is

$$
f_{M_{n}^{\prime}}(m)= \begin{cases}n(1-2|m|) & \text { for }|m| \leq 1 / 2, \\ 0 & \text { otherwise }\end{cases}
$$


from (2.5). Then the coverage probability is

$$
\begin{aligned}
P\left\{\left|M_{n}-\theta\right| \leq d\right\} & =P\left\{\left|M_{n}-\theta\right| / \xi \leq d / \xi\right\} \\
& =P\left\{\left|M_{n}^{\prime}\right| \leq d / \xi\right\} \\
& =\int_{-d / \xi}^{d / \xi} n(1-2|m|)^{n-1} d m \\
& =1-\left(1-\frac{2 d}{\xi}\right)^{n}
\end{aligned}
$$

for $0 \leq d \leq \xi / 2$. In order to satisfy $(2.13) \geq 1-\alpha$, we have to take

$$
n \geq \frac{\log \alpha}{\log (1-(2 d / \xi))}=: n^{*} \quad(\text { say })
$$

Here, we substitute the modified stopping rule (2.12) for the optimal fixed sample size $n^{*}$ since $n^{*} \simeq-\xi \log \alpha /(2 d)$ for small $d$. Then we have the following theorem.

Theorem 2.2. For the sequential estimation procedure $\left(\tau_{1},\left[M_{\tau_{1}}-d, M_{\tau_{1}}+\right.\right.$ $d]$ ), we have the following.

(i) $\lim _{d \rightarrow 0+} P\left\{\left|M_{\tau_{1}}-\theta\right| \leq d\right)=1-\alpha$ (i.e. asymptotic consistency).

(ii) $\tau_{1} / n^{*} \stackrel{\text { a.s. }}{\rightarrow} 1(d \rightarrow 0+)$.

(iii) $E\left(\tau_{1}\right) / n^{*} \rightarrow 1(d \rightarrow 0+)$.

Proof. (i) From Lemma 1 of Chow and Robbins (1965), the stopping rule $\tau_{1}$ given by (2.12) satisfies

$$
\lim _{d \rightarrow 0+}-\frac{2 d \tau_{1}}{\xi \log \alpha}=1 \quad \text { a.s. }
$$

Since $n\left(M_{n}-\theta\right)$ converges in distribution to a two-sided exponential distribution with density $\exp (-2|x|)$ as $n \rightarrow \infty$, it follows from Theorem 1 
of Anscombe (1952) that $\tau_{1}\left(M_{\tau_{1}}-\theta\right)$ converges in distribution to the same distribution as $d \rightarrow 0+$. Hence, since

$$
\frac{d \tau_{1}}{\xi} \stackrel{\text { a.s. }}{\rightarrow}-\frac{\log \alpha}{2} \quad \text { as } \quad d \rightarrow 0+
$$

from (2.14), it follows that

$$
\begin{aligned}
\lim _{d \rightarrow 0+} P\left\{\left|M_{\tau_{1}}-\theta\right| \leq d\right\} & =\lim _{d \rightarrow 0+} P\left\{\tau_{1}\left|M_{\tau_{1}}-\theta\right| / \xi \leq d \tau_{1} / \xi\right\} \\
& =\int_{(\log \alpha) / 2}^{-(\log \alpha) / 2} \exp (-2|x|) d x \\
& =1-\alpha .
\end{aligned}
$$

(ii) From (2.14) and L'Hopital's theorem, we have

$$
\frac{\tau_{1}}{n^{*}}=\frac{\tau_{1} \log \{1-(2 d / \xi)\}}{\log \alpha} \stackrel{\text { a.s. }}{\rightarrow} 1 \quad \text { as } \quad d \rightarrow 0+.
$$

(iii) Since, from Lemma 2 of Chow and Robbins (1965),

$$
\lim _{d \rightarrow 0+} \frac{2 d E\left(\tau_{1}-1\right)}{-\xi \log \alpha}=\lim _{d \rightarrow 0+} \frac{2 d E\left(\tau_{1}\right)}{-\xi \log \alpha}=1
$$

and

$$
d n^{*}=\frac{d \log \alpha}{\log \{1-(2 d / \xi)\}} \rightarrow \frac{\log \alpha}{-2 / \xi} \quad \text { as } \quad d \rightarrow 0+
$$

we have

$$
\lim _{d \rightarrow 0+} \frac{E\left(\tau_{1}\right)}{n^{*}}=\lim _{d \rightarrow 0+} \frac{d E\left(\tau_{1}\right)}{d n^{*}}=\frac{-(\xi \log \alpha) / 2}{(\log \alpha) /(-2 / \xi)}=1 .
$$

Thus we complete the proof.

\section{NUMERICAL EVALUATION}

In this section we examine the coverage probability of the procedure $\left[M_{\tau_{1}}-\right.$ $\left.d, M_{\tau_{1}}+d\right]$ by simulation based on 100000 repetitions. Since $M_{\tau_{1}}$ is location 
equivariant, we may assume $\theta=0$ without loss of generality. When $\alpha=$ $0.10, d=0.01(0.01) 0.05, \xi=1(1) 5$, Tables 1,2 and 3 show the values of coverage probabilities, the average sample numbers (ASNs) and the standard deviations (SDs) of the sequential estimation procedure $\left(\tau_{1},\left[M_{\tau_{1}}-d, M_{\tau_{1}}+d\right]\right)$ for $\alpha=0.10$, respectively. The result shows that we might need to improve the stopping rule, but provides us with a tentative information on $\theta$. Consider another stopping rule with the initial sample size as 10 instead of (2.12), that is,

$$
\tau_{1}^{\prime}=\inf \left\{\begin{array}{l|l}
n \geq 10 & \frac{R_{n}}{n-1} \leq-\frac{2 d}{\log \alpha}
\end{array}\right\} .
$$

Tables 4, 5 and 6 show the values of coverage probabilities, the ASNs and the SDs of the sequential estimation procedure $\left(\tau_{1}^{\prime},\left[M_{\tau_{1}^{\prime}}-d, M_{\tau_{1}^{\prime}}+d\right]\right)$ for $\alpha=0.10$, respectively. The result suggests that the estimation procedure is consistent for this case.

Table 1. Coverage probabilities of $\left[M_{\tau_{1}}-d, M_{\tau_{1}}+d\right]$ for $\alpha=0.10$

\begin{tabular}{|c|c|c|c|c|c|}
\hline$\xi \backslash d$ & 0.01 & 0.02 & 0.03 & 0.04 & 0.05 \\
\hline 1 & 0.7607 & 0.77045 & 0.77837 & 0.78468 & 0.79554 \\
\hline 2 & 0.75503 & 0.76211 & 0.76583 & 0.76831 & 0.77439 \\
\hline 3 & 0.75466 & 0.75862 & 0.76084 & 0.76462 & 0.76704 \\
\hline 4 & 0.75086 & 0.75763 & 0.76043 & 0.76246 & 0.76471 \\
\hline 5 & 0.75343 & 0.75475 & 0.75519 & 0.75825 & 0.75884 \\
\hline
\end{tabular}

Table 2. ASNs of $\tau_{1}$ for $\alpha=0.10$

\begin{tabular}{|c|c|c|c|c|c|}
\hline$\xi \backslash d$ & 0.01 & 0.02 & 0.03 & 0.04 & 0.05 \\
\hline 1 & 75.3458 & 37.9159 & 26.3105 & 19.5954 & 15.5133 \\
\hline 2 & 150.742 & 75.5505 & 51.2336 & 37.9784 & 30.5142 \\
\hline 3 & 225.222 & 112.968 & 75.4855 & 56.9635 & 45.3646 \\
\hline 4 & 300.76 & 150.42 & 101.419 & 75.4829 & 60.4096 \\
\hline 5 & 375.119 & 187.819 & 126.251 & 94.736 & 75.7516 \\
\hline
\end{tabular}


Table 3. SDs of $\tau_{1}^{\prime}$ for $\alpha=0.10$

\begin{tabular}{|c|c|c|c|c|c|}
\hline$\xi \backslash d$ & 0.01 & 0.02 & 0.03 & 0.04 & 0.05 \\
\hline 1 & 43.4924 & 21.8577 & 14.7446 & 11.0086 & 9.17156 \\
\hline 2 & 86.1962 & 43.4728 & 28.9139 & 21.8323 & 17.7156 \\
\hline 3 & 129.429 & 64.72 & 43.4497 & 32.6691 & 26.3042 \\
\hline 4 & 171.983 & 86.2782 & 57.6551 & 43.4353 & 34.8847 \\
\hline 5 & 214.641 & 107.455 & 71.7378 & 53.9417 & 43.4604 \\
\hline
\end{tabular}

Table 4. Coverage probabilities of $\left[M_{\tau_{1}^{\prime}}-d, M_{\tau_{1}^{\prime}}+d\right]$ for $\alpha=0.10$

\begin{tabular}{|c|c|c|c|c|c|}
\hline$\xi \backslash d$ & 0.01 & 0.02 & 0.03 & 0.04 & 0.05 \\
\hline 1 & 0.9093 & 0.9128 & 0.9217 & 0.9237 & 0.9265 \\
\hline 2 & 0.9051 & 0.9142 & 0.9108 & 0.9186 & 0.9235 \\
\hline 3 & 0.904 & 0.9022 & 0.907 & 0.9124 & 0.9066 \\
\hline 4 & 0.9006 & 0.9011 & 0.9133 & 0.9042 & 0.9119 \\
\hline 5 & 0.9059 & 0.9025 & 0.9002 & 0.9083 & 0.9084 \\
\hline
\end{tabular}

Table 5. ASNs of $\tau_{1}^{\prime}$ for $\alpha=0.10$

\begin{tabular}{|c|c|c|c|c|c|}
\hline$\xi \backslash d$ & 0.01 & 0.02 & 0.03 & 0.04 & 0.05 \\
\hline 1 & 116.997 & 58.995 & 39.990 & 29.975 & 24.940 \\
\hline 2 & 231.997 & 116.997 & 77.997 & 58.995 & 47.993 \\
\hline 3 & 346.998 & 173.998 & 116.998 & 87.997 & 70.997 \\
\hline 4 & 461.997 & 231.997 & 154.998 & 116.997 & 93.997 \\
\hline 5 & 576.997 & 288.997 & 192.998 & 144.999 & 116.997 \\
\hline
\end{tabular}

Table 6. SDs of $\tau_{1}^{\prime}$ for $\alpha=0.10$

\begin{tabular}{|c|c|c|c|c|c|}
\hline$\xi \backslash d$ & 0.01 & 0.02 & 0.03 & 0.04 & 0.05 \\
\hline 1 & 0.566183 & 0.518538 & 0.559546 & 0.689228 & 0.943005 \\
\hline 2 & 0.859796 & 0.586055 & 0.41362 & 0.482571 & 0.567398 \\
\hline 3 & 1.21506 & 0.655995 & 0.545588 & 0.461446 & 0.49555 \\
\hline 4 & 1.0107 & 0.702022 & 0.480908 & 0.513148 & 0.496942 \\
\hline 5 & 1.70099 & 1.18369 & 0.708751 & 0.539996 & 0.534994 \\
\hline
\end{tabular}




\section{SOME PROPERTIES OF THE ESTIMATION PROCEDURE}

Suppose that $X_{1}, X_{2}, \ldots, X_{n}, \ldots$ is a sequence of i.i.d. random variables according to the uniform distribution $[\theta-(\xi / 2), \theta+(\xi / 2)]\left(\theta \in \mathbb{R}^{1}, \xi>0\right)$. Let $X_{(1 \mid n)} \leq X_{(2 \mid n)} \leq \cdots \leq X_{(n \mid n)}$ be the order statistics of $X_{1}, X_{2}, \ldots, X_{n}(n \geq$ 1). Consider a stopping rule such that $\tau(A):=\min \left\{n \geq 2 \mid R_{n} /(n-1) \leq A\right\}$ for a positive constant $A$, and a sequential interval estimation procedure $\left[M_{\tau(A)}-d, M_{\tau(A)}+d\right]$ of $\theta$ for a constant $d(>0)$. In this section we consider some properties of $\left(\tau(A), M_{\tau(A)}\right)$. Hereafter we represent $P_{\theta, \xi}\{\cdot\}$ as $P\{\cdot\}$ for simplicity.

Theorem 4.1. The probability $P\{\tau(A)=n\}$ is given by

$$
P\{\tau(A)=n\}=p_{n-1}-p_{n} \quad(n \geq 2)
$$

recursively, where

$$
\begin{aligned}
& p_{1}:=P\{\tau(A)>1\}=1, \\
& p_{2}:=P\{\tau(A)>2\}= \begin{cases}\left(\frac{\xi-A}{\xi}\right)^{2} & \text { for } 0 \leq A \leq \xi, \\
0 & \text { otherwise }\end{cases} \\
& p_{n}:=P\{\tau(A)>n\} \\
& =\left\{\begin{array}{c}
\frac{(n-1)(n-2)}{\xi^{n-1}}\left[\left\{1-\frac{2}{\xi}(n-1) A\right\}\left(I_{n-1, n-3}-I_{n, n-3}\right)\right. \\
\left.+\frac{1}{\xi}\left(I_{n-1, n-2}-I_{n, n-2}\right)+I_{n, n-3}\right] \\
\cdot\left\{1-\left(\frac{(n-2) A}{\xi}\right)^{n-2}\left(n-1-\frac{(n-2)^{2} A}{\xi}\right)\right\}^{-1} p_{n-1} \\
\text { for } 0 \leq(n-1) A \leq \xi, \\
\text { otherwise, }
\end{array}\right.
\end{aligned}
$$

for $n \geq 3$, with

$$
I_{l, m}:=\frac{1}{(m+1)(m+2)}\left\{\xi^{m+2}+(m+1)((l-1) A)^{m+2}-\xi(m+2)((l-1) A)^{m+1}\right\}
$$


for $l \geq 1$ and $m \geq 0$.

Proof. First we have

$$
\begin{aligned}
P\{\tau(A)=n\}= & P\left\{R_{n} \leq(n-1) A, R_{i}>(i-1) A(i=2, \ldots, n-1)\right\} \\
= & P\left\{R_{i}>(i-1) A(i=2, \ldots, n-1)\right\} \\
& \quad-P\left\{R_{i}>(i-1) A(i=2, \ldots, n)\right\} \\
= & p_{n-1}-p_{n},
\end{aligned}
$$

where $p_{n}=P\left\{R_{i}>(i-1) A \quad(i=2, \ldots, n)\right\}(n \geq 1)$. Since $p_{2}=P\left\{R_{2}>\right.$ $A\}=P\left\{R_{2}^{\prime}>A / \xi\right\}$ and (2.6), it follows that $p_{2}=\{(\xi-A) / \xi\}^{2}$ for $0<A<$ $\xi$ and $p_{2}=0$ for $A \geq \xi$. We also have

$$
\begin{aligned}
p_{n} & =P\left\{R_{n}>(n-1) A \mid R_{i}>(i-1) A(i=2, \ldots, n-1)\right\} p_{n-1} \\
& =P\left\{R_{n}>(n-1) A \mid R_{n-1}>(n-2) A\right\} p_{n-1} \\
& =\frac{P\left\{R_{n}>(n-1) A, R_{n-1}>(n-2) A\right\}}{P\left\{R_{n-1}>(n-2) A\right\}} p_{n-1} .
\end{aligned}
$$

The denominator of (4.1) is

$$
P\left\{R_{n-1}>(n-2) A\right\}=1-\left(\frac{n-2}{\xi} A\right)^{n-2}\left(n-1-\frac{(n-2)^{2}}{\xi} A\right)
$$

for $0<(n-2) A / \xi<1$, since $P\left\{R_{n-1}>(n-2) A\right\}=P\left\{R_{n}^{\prime}>(n-2) A / \xi\right\}$ and (2.6), and $P\left\{R_{n-1}>(n-2) A\right\}=0$ for $(n-2) A / \xi \geq 1$. Denoting by $f_{X_{(1 \mid n-1)}, X_{(n-1 \mid n-1)}}(u, v)$ the joint density of $X_{(1 \mid n-1)}$ and $X_{(n-1 \mid n-1)}$, we have

$$
\begin{aligned}
& P\left\{R_{n}>(n-1) A, R_{n-1}>(n-2) A\right\} \\
= & \iint_{B_{n-1}} P\left\{R_{n}>(n-1) A \mid X_{(1 \mid n-1)}=u, X_{(n-1 \mid n-1)}=v\right\} \\
& \cdot f_{X_{(1, \mid n-1)}, X_{(n-1 \mid n-1)}}(u, v) d u d v \\
= & \left(\iint_{B_{n-1}-B_{n}}+\iint_{B_{n}}\right) P\left\{R_{n}>(n-1) A \mid X_{(1 \mid n-1)}=u, X_{(n-1 \mid n-1)}=v\right\} \\
& \cdot f_{X_{(1 \mid n-1)}, X_{(n-1 \mid n-1)}}(u, v) d u d v
\end{aligned}
$$


where $B_{i}:=\{(u, v) \mid v-u>(i-1) A, \theta-(\xi / 2) \leq u \leq v \leq \theta+(\xi / 2)\}(i \geq 1)$. If $\left(X_{(1 \mid n-1)}, X_{(n-1 \mid n-1)}\right)=(u, v)$ is given for $\theta-(\xi / 2) \leq u \leq v \leq \theta+(\xi / 2)$, then

$$
\left(X_{(1 \mid n)}, X_{(n \mid n)}\right)= \begin{cases}\left(X_{n}, v\right) & \text { with probability } F(u) \\ (u, v) & \text { with probability } F(v)-F(u) \\ \left(u, X_{n}\right) & \text { with probability } 1-F(v)\end{cases}
$$

where $F(\cdot)$ is the distribution function of $X_{i}$ given by

$$
F(x)= \begin{cases}0 & \text { if } \quad x<\theta-(\xi / 2) \\ \{x-\theta+(\xi / 2)\} / \xi & \text { if } \theta-(\xi / 2) \leq x \leq \theta+(\xi / 2) \\ 1 & \text { if } x>\theta+(\xi / 2)\end{cases}
$$

So, $X_{(n \mid n)}>X_{(1 \mid n)}+(n-1) A$ holds with probability 1 given $(u, v)$ satisfying $v>u+(n-1) A$, and with probability $\{1+F(v-(n-1) A)-F(u+(n-1) A)\}$ given $(u, v)$ satisfying $u+(n-2) A<v<u+(n-1) A$. Then the right-hand side of (4.2) is equal to

$$
\begin{aligned}
& \iint_{B_{n-1}-B_{n}}\{1+F(v-(n-1) A)-F(u+(n-1) A)\} \\
& \quad \cdot f_{X_{(1 \mid n-1)}, X_{(n-1, \mid n-1)}}(u, v) d u d v \\
& +\iint_{B_{n}} f_{X_{(1 \mid n-1)}, X_{(n-1 \mid n-1)}}(u, v) d u d v .
\end{aligned}
$$

Since

$$
f_{X_{(1 \mid n-1)}, X_{(n-1 \mid n-1)}}(u, v)=\left\{\begin{array}{l}
(n-1)(n-2)\{F(v)-F(u)\}^{n-3} / \xi^{2} \\
\text { for } \theta-(\xi / 2) \leq u \leq v \leq \theta+(\xi / 2), \\
0 \quad \text { otherwise }
\end{array}\right.
$$

it follows from (4.3) that (4.4) equals, by letting $I_{l, m}:=\iint_{B_{l}}(v-u)^{m} d u d v$ for $l \geq 1$ and $m \geq 0$,

$$
\begin{aligned}
\frac{(n-1)(n-2)}{\xi^{n-1}}\left[\left\{1-\frac{2}{\xi}(n-1) A\right\}\left(I_{n-1, n-3}-I_{n, n-3}\right)\right. & \\
+ & \left.\frac{1}{\xi}\left(I_{n-1, n-2}-I_{n, n-2}\right)+I_{n, n-3}\right] \quad(n \geq 3)
\end{aligned}
$$


with $I_{l, m}=\frac{1}{(m+1)(m+2)}\left\{\xi^{m+2}+(m+1)((l-1) A)^{m+2}-\xi(m+2)((l-1) A)^{m+1}\right\}$, which is the desired equality.

Remark. The values of ASN $E(\tau(A))$ can be calculated by using $\left\{p_{n}\right\}$. Indeed, since $p_{n}=P\{\tau(A)>n\}(n \geq 2)$,

$$
\begin{aligned}
\sum_{i=2}^{\infty} p_{i} & =\sum_{i=2}^{\infty} P\{\tau(A)>i\}=\sum_{i=2}^{\infty} \sum_{j=i+1}^{\infty} P\{\tau(A)=j\}=\sum_{j=3}^{\infty} \sum_{i=2}^{j-1} P\{\tau(A)=j\} \\
& =\sum_{j=3}^{\infty} j P\{\tau(A)=j\}-2 \sum_{j=3}^{\infty} P\{\tau(A)=j\} \\
& =E(\tau(A))-2 P\{\tau(A)=2\}-2\{1-P\{\tau(A)=2\}\} \\
& =E(\tau(A))-2,
\end{aligned}
$$

we have

$$
E(\tau(A))=\sum_{i=2}^{\infty} p_{i}+2
$$

\section{ACKNOWLEDGEMENTS}

The authors would like to thank the referees for their valuable comments.

\section{$\underline{\text { REFERENCES }}$}

Akahira, M. (1993). Two-stage sequential estimation procedures for the uniform distribution. (In Japanese). Proc. Sympos., Res. Inst. Math. Sci., Kyoto University, 842, 151-156.

Anscombe, F. J. (1952). Large-sample theory of sequential estimation. Proc. Cambridge Philos. Soc., 48, 600-607.

Bose, A. (2001). A boundary crossing problem with application to sequential estimation. Sequential Analysis, 20, 65-76. 
Chow, Y. S. and Robbins, H. (1965). On the asymptotic theory of fixedwidth sequential confidence intervals for the mean. Ann. Math. Statist., 36, 457-462.

Cooke, P. J. (1971). Sequential estimation in the uniform density. J. Amer. Statist. Assoc., 66, 614-617.

Ghosh, M., Mukhopadhyay, N. and Sen, P. K. (1997). Sequential Estimation. Wiley, New York.

Graybill, F. A. and Connell, T. L. (1964). Sample size required to estimate the parameter in the uniform density within $d$ units of the true value. J. Amer. Statist. Assoc., 59, 550-560.

Perruzzi, J. J. and Hilliard, E. J. (1984). Modeling time-delay measurement errors using a generalized beta density function. J. Acoustical Society of America 75, 197-201

Mukhopadhyay, N. and Cicconetti, G. (2002). Second-order properties of a two-stage point estimation procedure for the range in a Power family distribution. Calcutta Statist. Assoc. Bull., 52, 219-234.

Wald, A. (1950). Statistical Decision Functions. Wiley, New York.

Zimmels, Y. (1983). Theory of kindered sedimentation of polydisperse mixtures. American Institute of Chemical Engineers Journal, 29, 669-676. 